\title{
Nonlinear Microwave Properties of HTS Thin Film Coplanar Devices
}

\author{
P. Lahl and R. Wördenweber
}

\begin{abstract}
It is demonstrated that the combination of vortex matter and rf measurements yields new insight into the microwave properties of superconducting thin film devices, both in small magnetic fields and zero field. The comparison of field-cooled and different types of field sweep experiments on coplanar high- $T_{c}$ thin film resonators shows that the microwave properties strongly depend on magnetization and vortex distribution in the superconducting film as well as on the way, the magnetic field is approached. Thus, using vortices as a kind of local probe for the microwave properties leads to a consistent explanation of the microwave power handling in nonzero and zero magnetic field. In a model that is derived from the experiments, the nonlinear microwave behavior is explained by the limitation of the total current density in the device considering contributions of the rf field and the screening of the magnetic field and vortices to the current. The limiting current value seems to be related to the dc critical current of the superconductor.
\end{abstract}

Index Terms-Nonlinear microwave properties, superconducting films, vortex matter.

\section{INTRODUCTION}

$\mathbf{T}$ HE use of superconducting thin films in microwave devices allows a dramatic reduction of the device dimension at comparable or higher performance with respect to conventional nonsuperconducting devices [1]. As a consequence, larger power densities are encountered in these devices. However, in contrast to their normal-conducting competitors, the power handling capability of superconducting microwave devices is limited by the onset of nonlinear effects in form of a strong increase of the dissipation or intermodulation products at large rf power. Although various physical origins are discussed (e.g., weak links in granular superconductors [2], pair breaking [3], thermal effects [4], magnetic effects [5]), the physical mechanism, that is responsible for the nonlinear surface resistance and that represents a serious restriction for a number of rf applications, is not fully understood. In this paper, we demonstrate that the combination of vortex matter and microwave properties leads to new insight into the nature of the nonlinear microwave properties of superconducting material.

\section{EXPERImental SetuP AND TeChNiQue}

Coplanar thin film resonators (resonant frequency of $f=$ 1.4 GHz) are patterned from epitaxial YBCO films (thickness $d \approx 300 \mathrm{~nm}$ ) on sapphire substrates. The width of the cen-

Manuscript received August 6, 2002. This work was supported by DFG Grant no. WO549/3-1 and ESF scientific program VORTEX.

The authors are with Forschungszentrum Jülich, ISG, 52425 Jülich, Germany (e-mail: p.lahl@fz-juelich.de)

Digital Object Identifier 10.1109/TASC.2003.812046

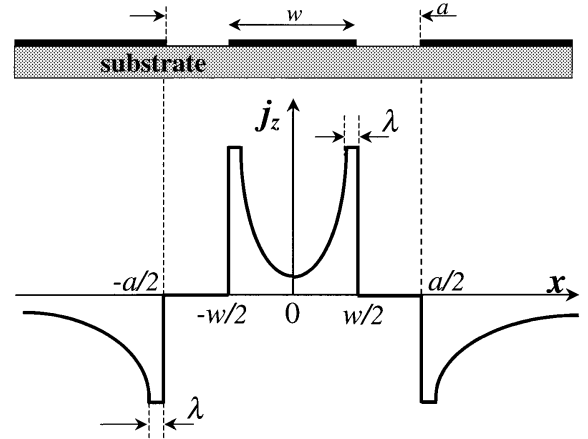

Fig. 1. Schematic sketch of the design and microwave current distribution of a superconducting thin-film coplanar resonator.

tral conductor is $w=100 \mu \mathrm{m}$ and the distance of the groundplanes is $a=182 \mu \mathrm{m}$ yielding a transmission line impedance $Z_{L}=50 \Omega$. The $1 \mathrm{D}$ current density distribution across the center conductor of coplanar microwave devices can be approximated by [6]

$$
\begin{aligned}
j_{z}(x)= & \frac{I}{w \cdot K\left(\frac{w}{a}\right)} \\
& \cdot\left[\begin{array}{c}
{\left[\left(1-\left(\frac{2 x}{w}\right)^{2}\right) \cdot\left(1-\left(\frac{2 x}{a}\right)^{2}\right)\right]^{-1 / 2} \text { for }|x|<\frac{w}{2}-\lambda} \\
{\left[\left(1-\left(\frac{w}{a}\right)^{2}\right) \cdot \frac{\lambda}{w}\right]^{-1 / 2} \text { for } \frac{w}{2}-\lambda<|x|<\frac{w}{2}}
\end{array}\right.
\end{aligned}
$$

Here, $\lambda$ is the London penetration depth and $K(w / a)$ the complete elliptic integral which for our geometry is $K \approx 1.72$. The maximum current density in the groundplane is at least by a factor of $(w / a)^{1 / 2}$ smaller than in the central conductor. Thus the nonlinear rf properties are considered to be dominated by the central conductor.

Due to this characteristic current distribution (see Fig. 1), first the power handling capability is relatively small so that thermal effects are limited to a temperature increase of $\Delta T<15 \mu \mathrm{K}$ for the highest occuring rf powers in the experiments and thus can be neglected [7], [12]. Second, it allows to locally investigate the microwave properties by applying small magnetic fields. Since the rf current is strongly peaked at the edge of the central conductor (see Fig. 1), these devices turn out to be highly sensitive to magnetization or vortex penetration. Furthermore, by establishing different vortex distributions in the resonator, the impact of vortices on the microwave properties can be analyzed locally. Differences in the resulting quality factor $\mathrm{Q}$ and the power handling capability of the resonator for different vortex distributions reveal new insight in the nature of the nonlinear rf properties of superconducting material. Thus, the specific properties 


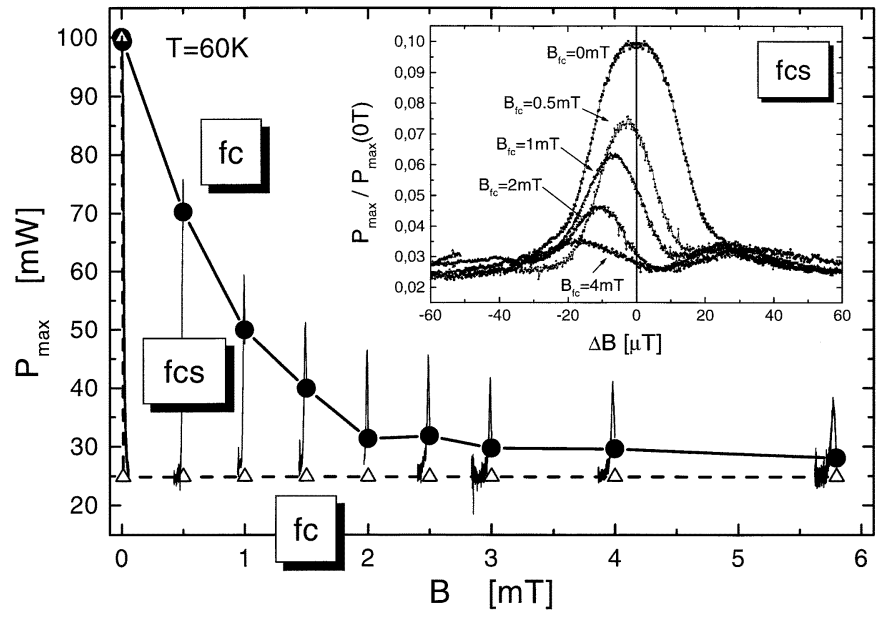

Fig. 2. Field dependence of the power handling capability of a coplanar YBCO resonator for field-cooled (fc, solid circles), zero-field cooled (zfc, open triangles) and field-cooled sweep (fcs, solid lines) experiments. The inset shows the fcs measurement starting a different fields $B_{\mathrm{fc}}$ on an enlarged and normalized scale.

of coplanar microwave devices (i.e., small power handling capability and extremely inhomogeneous current distribution) are ideal for the investigation of the mechanism that limits the power handling capability.

The power handling capability is characterized by the oscillating if power $P_{\max }$ at which the unloaded quality factor $Q_{0}$ is degraded to $80 \%$ of its low-power value, i.e., $Q_{0}\left(P_{\max }\right)=$ $0.8 \cdot Q_{0}(P \rightarrow 0)$ and $Q_{0} \approx 20.000-30.000$ for $P \rightarrow 0$ and $T \rightarrow 0$. In field cooled (fc) and zero field cooled (zfc) experiments the resonator is cooled to the superconducting state in an applied magnetic field or in zero field, respectively. In field cooled sweep (fcs) experiments, the sample is first cooled to the superconducting state in an applied field $B_{\mathrm{fc}}$ (where the field cooled state is established) and subsequently the field is modified by $\Delta B$.

\section{DISCUSSION OF THE RESUlTS}

Fig. 2 represents an overview of the field dependence of the power handling capability of a typical coplanar resonator. The power handling capability $P_{\max }$ depends strongly upon the way the magnetic field is established. Whereas fc measurements show a gradual decrease of $P_{\max }$, in zfc experiments a strong decrease of the power handling capability within a few $\mu \mathrm{T}$ is followed by an almost constant $P_{\max }$. The different behavior seems to be a consequence of the vortex distribution in the resonator. A homogeneous vortex distribution in the superconductor is expected for fc experiments. The linear increase of the vortex density with increasing field seems to lead to the gradual decrease of $P_{\max }$. In contrast to the fc experiments, an inhomogeneous vortex distribution is expected for the zfc case. Due to the vortex penetration into the field-free superconductor in the zfc case, the position of the largest vortex density coincides with the maximum of the rf current at the edge of the central conductor. This explains the strong reduction of $P_{\max }$ already at extremely small fields of a few $\mu \mathrm{T}$. A further increase of the field in the zfc case does not affect

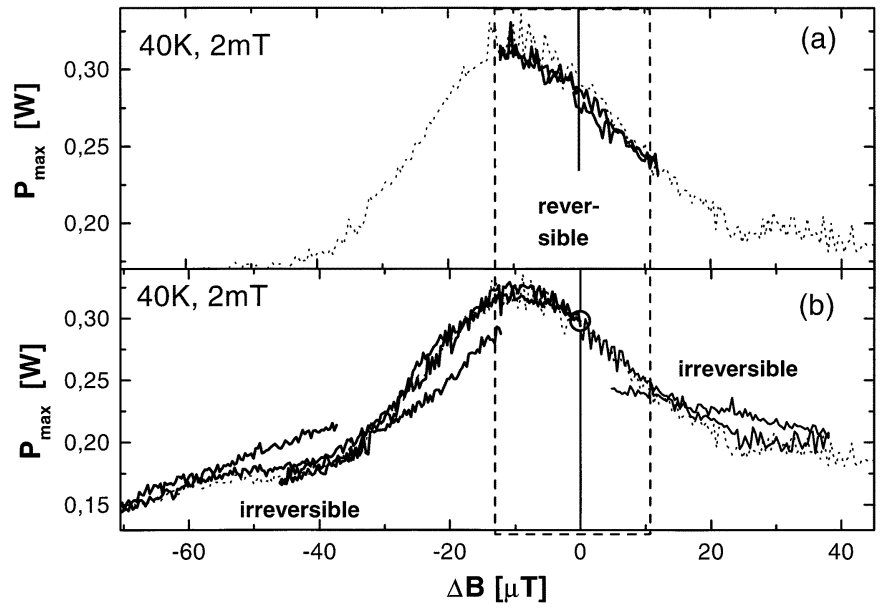

Fig. 3. Magnetic field cycles with different amplitudes in fcs experiments reveal the underlying mechanism of magnetization (reversible behavior in (a)) and vortex or antivortex penetration (irreversible behavior in (b)). The gray line represents the single scan (see inset Fig. 2) starting at $B_{\mathrm{fc}}=2 \mathrm{mT}$ (open circle).

the power-handling capability seriously. This is consistent with other observations of flux penetration at small fields, where an increase of the magnetic field first leads only to a shift of the flux front into the superconductor [8]. Since the interior of the superconductor does not carry large rf currents, this shift does not affect the power handling capability seriously. Furthermore, the fc and zfc values of $P_{\max }$ seem to merge at large fields. This is expected for the field, for which the zfc flux front approaches the center of the sample. For these fields similar vortex densities are expected at the edge of the superconductor for the fc and zfc case.

The most interesting features, that also confirm the interpretation of the fc and zfc data given above, are observed for fcs measurements (inset of Fig. 2 and for field cycles Fig. 3). Starting with the fc value, fcs experiments show a characteristic variation of $P_{\max }$ with increasing and decreasing field, which provides an ideal fingerprint of magnetization and vortex penetration. For finite starting fields $B_{\mathrm{fc}}$, a nonsymmetrical $\Delta B$-dependence of $P_{\max }$ is obtained. For $B_{\mathrm{fc}}>0$, first $P_{\max }$ increases for decreasing field and decreases for increasing field, then for larger field changes $P_{\max }$ decreases for decreasing field and shows a characteristic small peak for increasing field. The position of the maximum of $P_{\max }(\Delta B)$ for $\Delta B<0$ decreases with increasing field $B_{\mathrm{fc}}$.

Field cycles with different amplitude (Fig. 3) prove the different underlying mechanisms. For small field amplitudes $\Delta B$, a reversible magnetization is observed. The magnetization leads to a decrease of $P_{\max }$ for $\Delta B>0$. For $\Delta B<0$ a compensation of $B_{\mathrm{fc}}$ causes an increase of $P_{\max }$. Modification of $P_{\max }$ by up to $50 \%$ take place within a few $\mu \mathrm{T}$. Only for larger amplitudes $|\Delta B|>10 \mu \mathrm{T}$ vortices $(\Delta B>0)$ or antivortices $(\Delta B<0)$ start to nucleate and penetrate the resonator structure. This leads to an irreversible modification of $P_{\max }$ (see Fig. 3(b)). The penetration of the first vortices is accompanied by a small increase of the $P_{\max }$ for the positive branch, that can be interpreted by a relaxation of the magnetization at the edge of the superconducting central conductor. Obviously this effect is not possible 
for the negative branch, here $P_{\max }$ decreases when the first antivortices penetrate.

\section{Modelling OF THE Results}

In the following, the field-dependence of $P_{\max }$ will be discussed in terms of a new model that considers (i) a superposition and (ii) limitation of current density in the superconductor. Ambient magnetic fields lead to screening currents in the superconductor. The resulting screening current densities ( $j_{M}$ due to a magnetization and $j_{V}$ due to vortices) superimpose the rf current density $j_{\mathrm{rf}}$, yielding a total current density $j_{\text {tot }}=$ $j_{\mathrm{rf}}+j_{M}+j_{V}$ in the coplanar resonator. The nonlinearity implies that the total current density is limited by a critical value, i.e., $j_{\text {tot }}<j_{\max }$ and thus the linear behavior is restricted to the regime $j_{\mathrm{rf}}<j_{\max }$. Assuming a field dependence of the limiting current [12]

$$
j_{\max }(B, x)=j_{\max , B=0}(x)-j_{M}(B, x)-j_{V}(B, x)
$$

this model automatically yields a power handling capability that strongly depends on the kind of experiment.

\section{A. Field Cooled Experiments}

In $\mathrm{fc}$ experiments a homogeneous distribution of vortices in the superconductor can be assumed and magnetization effects can be neglected for not too small fields, i.e., $j_{\text {tot }} \approx j_{\mathrm{rf}}+j_{V}$. Assuming an ideal vortex lattice, the positions of the vortices in the central conductor of the resonator is given in coordinates of Fig. 1 by $\left|x_{n}\right|=\left[w-(2 \cdot \gamma \cdot n+1) \cdot a_{0}\right] / 2$ with $n=0,1,2, \ldots, a_{0}=\left(\phi_{o} / \gamma B\right)^{1 / 2}$ and $\gamma=1$ or 0.866 for a quadratic or hexagonal vortex lattice, respectively. Since the screening current drops exponentially with the characteristic length $\lambda$ (London penetration length), we assume, that the total current density in the regime $x_{n} \pm \alpha \lambda$ of the cross-section is dominated by the contribution of the vortex screening current with $\alpha \approx 0.5-1$. Thus, the maximum total current $I_{\text {tot,max }}$ can be obtained by summation of the maximum rf current and the redistributed current due to the screening current of vortices at positions $x_{n}$ :

$$
I_{\mathrm{tot}, \max }=I_{\mathrm{rf}, \max }+\sum_{\text {Vortices } n} j_{z}\left(x_{n}\right) \cdot 2 \lambda_{\mathrm{eff}} \cdot \alpha .
$$

The maximum rf current can be derived from the measurement of $P_{\max }$ according to $I_{\mathrm{rf}, \max }=\left(2 P_{\max } / Z_{L}\right)^{1 / 2}$ with the line impedance $Z_{L}=50 \Omega$. Using (1) yields the maximum total current:

$$
\begin{aligned}
& I_{\text {tot, } \max }=I_{\text {rf. } \max } \\
& \cdot\left(1+\frac{\lambda_{\text {eff }} \cdot \alpha}{w \cdot K\left(\frac{w}{a}\right)} \sum_{\text {Vortices } n} \frac{2}{\sqrt{\left[1-\left(\frac{2 \cdot x_{n}}{w}\right)^{2}\right] \cdot\left[1-\left(\frac{2 \cdot x_{n}}{a}\right)^{2}\right]}}\right) .
\end{aligned}
$$

Fig. 4(a) shows the field dependence of the maximum total current for a set of fc experiments on a typical resonator. The transformation of the rf current via (4) to the total current demonstrates the expected field independence of $I_{\text {tot, } \max }((2))$ for a large magnetic field range, i.e., between $0.5 \mathrm{mT}$ and $3 \mathrm{mT}$.
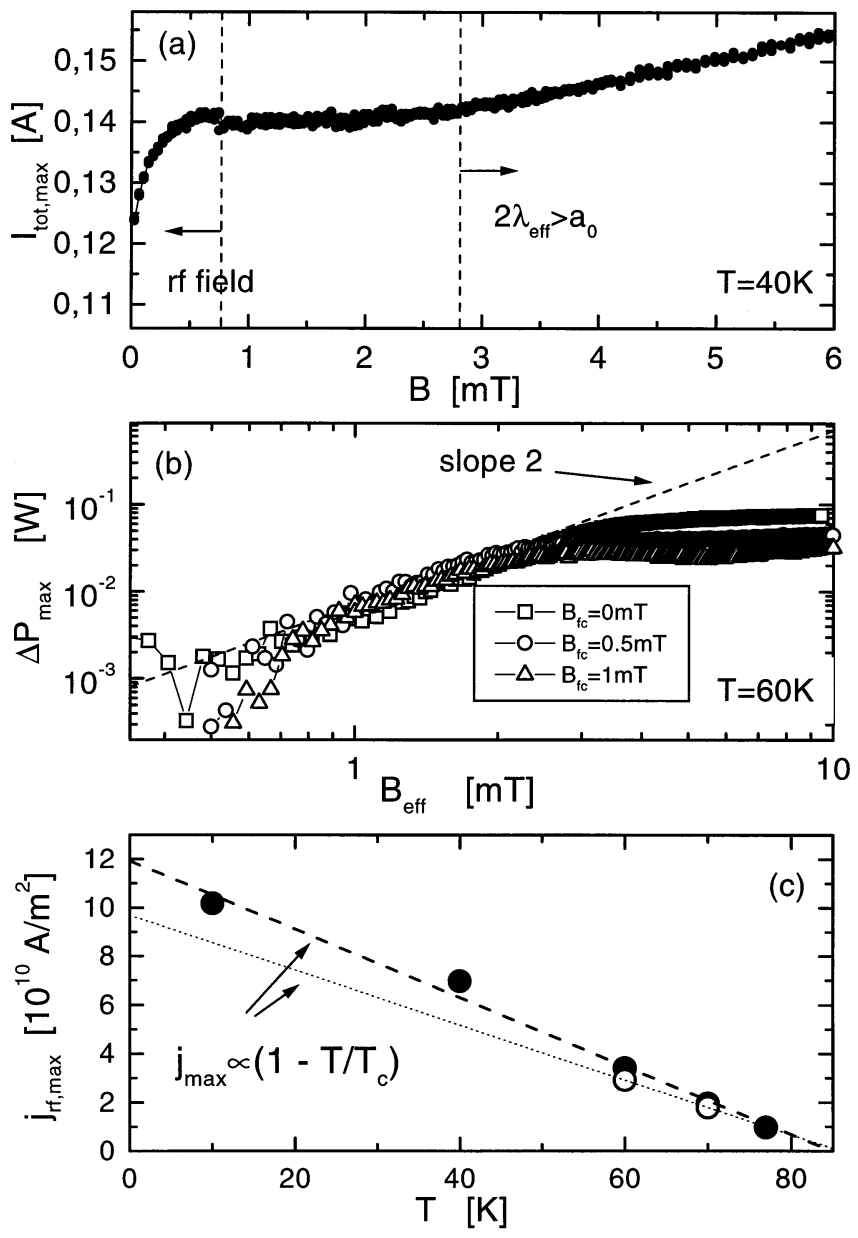

Fig. 4. (a) Field dependence of the calculated total current ((4)) for a typical resonator. The values for the rf self-field and the onset of overlap and compensation of vortex screening currents are marked and restrict the validity of (4) and, thus, regime of constant total current. (b) $\Delta P_{\max }$ as function of effective magnetic field for $\mathrm{zfc}$ and fcs experiments for different values of the field cooled magnetic field $B_{\mathrm{fc}}$. (c) Temperature dependence of the maximum rf current density for two resonators patterned of YBCO films of different quality (solid symbols: optimized film quality; open symbol: oxygen depleted YBCO film).

The deviation from the field independence at small and large fields can be explained by the effect of magnetization due to the rf field and the overlap of vortices, respectively. Both effects are not accounted for in (4). For $B<0.5 \mathrm{mT}$ the calculated total current decreases slightly with decreasing field. Since residual fields (e.g., earth field) have been compensated in the experimental setup, we consider the rf field to be responsible for the deviation. The rf magnetic field $B_{\mathrm{rf}}$ can be approximated by Ampere's law yielding $B_{\mathrm{rf}} \approx \mu_{0} I_{\mathrm{rf}} / 2 w \approx 0.88 \mathrm{mT}$ using the experimental value $I_{\mathrm{rf}} \approx 0.14 \mathrm{~A}$. This is of the order of the field up to which the deviation from $I_{\text {tot,max }}=$ constant is experimentally observed in the small field regime. For fields $B>3 \mathrm{mT} I_{\text {tot,max }}$ increases with increasing field. In (3) vortices are assumed to be independent. However, for $a_{0} \leq 2 \lambda_{\text {eff }}$ vortices start to overlap and, thus, the screening currents of adjacent vortices start to partially cancel each other. Inserting reasonable values for the effective penetration depth yields field values $B \geq 3 \mathrm{mT}$ for which the overlap of vortices leads to an overestimation of the total current in (4). 


\section{B. Zero Field Cooled and Field Cooled Sweep Experiments}

In contrast to fc experiments, in $\mathrm{zfc}$ and fcs experiments field changes introduce a magnetization of the superconductor and, subsequently, gradients in the vortex density. However, restricting our consideration to small field changes, penetration of vortices (additional to those that are frozen in during cooling in the field $B_{\mathrm{fc}}$ ) can be neglected and, thus, $\Delta j_{\text {tot }} \approx \Delta j_{\mathrm{rf}}+\Delta_{\mathrm{jM}}=0$ is expected. Let us consider the case of zfc and fcs experiments for increasing field. The effective field drops exponentially at the superconductor surface, i.e., $B\left(x^{\prime}\right)=B_{\text {eff }} \cdot \exp \left(-x^{\prime} / \lambda\right)$ with $x^{\prime}=|x \pm w / 2|$. The resulting screening current density is given by $j_{M}\left(x^{\prime}\right)=\left(B_{\text {eff }} / \lambda \mu_{0}\right) \exp \left(-x^{\prime} / \lambda\right)$, the total screening current can be approximated in analogy to the description of the skin effect by $j_{M}(0) \cdot \lambda \cdot t \propto B_{\text {eff }}$ where $t$ represents the film thickness. Thus, the change of the power handling capability can be approximated by:

$$
\begin{aligned}
\Delta P_{\max }=P_{\max }\left(B_{\text {eff }}\right)-P_{\max } & \left(B_{\mathrm{fc}}\right) \\
& \propto\left(j_{M}(0) \cdot \lambda_{\text {eff }} \cdot t\right)^{2} \propto B_{\text {eff }}^{2}
\end{aligned}
$$

Furthermore, the effective field at the superconductor surface is given by $B_{\mathrm{eff}}=B_{\mathrm{fc}}+s \cdot \Delta B$, where $s=(1-D)^{-1}$ is the field enhancement due to the demagnetizing factor $D \cdot D=$ 0.99456 is derived from the fcs experiments for the geometry of our samples by assuming, that the maximum power handling capability is achieved for field compensation at the edge of the superconductor, i.e., $B_{\mathrm{fc}}+s \cdot \Delta B=0$ at the maximum of $P_{\max }$ in fcs experiments (inset of Fig. 2) [12].

The double-logarithmic plot (Fig. 4(b)) of $\Delta P_{\max }$ as a function of effective magnetic field $B_{\text {eff }}$ for a typical set of $\mathrm{zfc}$ and fcs experiments demonstrates the excellent agreement of the data with the prediction in (5). $\Delta P_{\max }$ increases quadratically with $B_{\text {eff }}$ and, even more, all data (zfc and fcs for different fields $B_{\mathrm{fc}}$ ) fall on one line. A deviation from the quadratic dependence is observed at higher fields starting for all curves at $B_{\text {eff }} \approx 2 \mathrm{mT}$. This deviation has to be ascribed to the effect of the geometrical barrier for collective vortex entry [9]. For the geometry of the resonator, the geometrical field changes barrier for flux penetration is $B_{\mathrm{cp}} \approx 15 \mu \mathrm{T}$ which corresponds to an effective field of $2.8 \mathrm{mT}$. Therefore, for effective fields larger than $2.8 \mathrm{mT}$ vortices are energetically stable inside the superconductor. This leads to an additional contribution to the current in the superconductor and, thus, a reduced increase in the experimentally observed $\Delta P_{\max }$.

\section{Zero Field Experiments}

Finally, zero-field measurements are discussed in terms of our model. Generally the rf current density profile shows a maximum at the edges of the superconducting resonator. According to (1) the maximum current density is given by

$$
j_{\mathrm{rf}, \max }=\frac{\sqrt{2 P_{\max }}}{K\left(\frac{w}{a}\right)}\left[Z \cdot \lambda_{\text {eff }} w t^{2}\left(1-\left(\frac{w}{a}\right)^{2}\right)\right]^{-1 / 2} .
$$

Thus, the maximum current density in the superconductor can be derived from experimental values of $P_{\max }$. Fig. 4(c) shows the resulting temperature dependence of $j_{\mathrm{rf} \text {, max }}$ for two resonators patterned from YBCO films of different quality. Two interesting aspects should be noticed, i.e., $j_{\mathrm{rf} \text { max }}$ increases linear with temperature and the order of magnitude is $j_{\mathrm{rf}, \max }(77 \mathrm{~K}) \approx 10^{10} \mathrm{Am}^{-2}$ and $j_{\mathrm{rf}, \max }(10 \mathrm{~K}) \approx 10^{11} \mathrm{Am}^{-2}$. Both, temperature dependence and magnitude of the maximum rf current density agree with the dc critical current for typical YBCO thin films [10]. This suggests, that the nonlinear microwave behavior is strongly related to the dc critical current of the superconductor. A relations between microwave power handling and pair-breaking mechanisms [11] could not be verified by our experiments.

\section{CONCLUSIONS}

In conclusion, we have demonstrated that the use of vortex matter in microwave experiments yields new insight into the microwave properties of superconducting thin films. The comparison of the microwave power handling capability of coplanar HTS thin film resonators in fc, zfc and fcs experiments shows, that (i) the microwave properties depend on magnetization and vortex distribution, that (ii) power handling and quality factor of the devices are strongly affected by the way the magnetic field is approached, and that (iii) the microwave power handling can be understood in terms of a simple model, that considers all contributions to the current in the superconductor (i.e., rf and screening currents) which seems to be limited by the dc critical current. The model explains the microwave behavior in nonzero and zero magnetic field. Thus, magnetization and vortex penetration can be used to probe the local microwave properties, and reveal the mechanism of nonlinear microwave properties. However, it also bears new and interesting aspects of vortex physics in patterned superconducting thin film devices.

\section{REFERENCES}

[1] H. J. Chaloupka, Microwave Application of High Temperature Superconductors, H. Weinstock, Ed. Dordrecht: Kluwer, 1999, NATO ASI Series.

[2] D. E. Oates, P. P. Nguyen, G. Dresselhaus, M. S. Dresselhaus, G. Koren, and E. Polturak, J. Supercond., vol. 8, p. 725, 1995.

[3] R. B. Hammond, R. R. Soares, B. A. Willemsen, T. Dahm, D. J. Scalapino, and J. R. Schrieffer, J. Appl. Phys., vol. 84, p. 5662, 1998.

[4] J. Wosik, L. M. Xie, K. Nesteruk, D. Li, J. H. Miller Jr, and S. A. Long, IEEE Trans. on Appl. Supercond., vol. 7, p. 1470, 1997.

[5] M. A. Hein, Supercond. Sci. Technol., vol. 10, p. 867, 1997.

[6] I. G. Vendik, O. G. Vendik, and D. I. Kaparkov, "Superconductor Microwave Technology Part II: Superconducting Microwave Circuits,", ISSN 1103-4599, ISRN CTH-MVT-R-24-SE, 1996.

[7] P. Lahl, R. Wördenweber, and M. Hein, Appl. Phys. Lett., vol. 79, 2001.

[8] M. Pannetier, R. J. Wijngaarden, I. Fløan, J. Rector, R. Griessen, P. Lahl, and R. Wördenweber, submitted for publication.

[9] E. Zeldov, A. I. Larkin, V. B. Geshkenbein, M. Koncykowski, D. Majer, B. Khaykovich, V. M. Vinokur, and H. Shtrikman, Phys. Rev. Lett., vol. 73, p. $1428,1994$.

[10] B. Dam, J. M. Huibregtse, F. C. Klaassen, R. C. F. Van der Geest, G. Doornbos, J. H. Rector, A. M. Testa, S. Freisem, J. C. Martinez, B. Stäuble-Pümpin, and R. Griessen, Nature, vol. 399, p. 439, 1999.

[11] T. Dahm, D. J. Scalapino, and B. A. Willemsen, J. Supercond., vol. 12, 1999.

[12] P. Lahl, "Verlag Forschungszentrum Jülich GmbH - Zentralbibliothek," Ph.D., Jülich, Germany, 2001. 